\title{
Archéopages
}

Archéopages

Archéologie et société

34 | 07/2012

Campagnes

\section{Deux exemples de modélisation en 3D de sites urbains}

\section{Mehdi Belarbi}

\section{(2) OpenEdition}

1 Journals

Édition électronique

URL : https://journals.openedition.org/archeopages/420

DOI : 10.4000/archeopages.420

ISSN : 2269-9872

\section{Éditeur}

INRAP - Institut national de recherches archéologiques préventives

\section{Édition imprimée}

Date de publication : 1 février 2012

Pagination : $91-93$

ISSN : 1622-8545

\section{Référence électronique}

Mehdi Belarbi, «Deux exemples de modélisation en 3D de sites urbains », Archéopages [En ligne], 34 |

07/2012, mis en ligne le 01 juillet 2012, consulté le 22 janvier 2022. URL : http://

journals.openedition.org/archeopages/420 ; DOI : https://doi.org/10.4000/archeopages.420 
de la fouille à un moment donné. La souplesse et le faible investissement de la méthode permettent d'envisager plusieurs relevés par opération. Ils ont pour fonction leur mise à plat afin d'en extraire des plans et des coupes, mais ils peuvent également servir de support à la mémoire. Cette fonction est loin d'être négligeable dans ces contextes où l'enchevêtrement des faits rend leurs lectures difficiles. Comme pour le passage du noir et blanc à la couleur ou de l'argentique au numérique, la $3 \mathrm{D}$ constitue une nouvelle étape dans l'évolution de la documentation. Elle n'a pas pour vocation d'être systématiquement publiée sur papier et peut servir d'archive. La $3 \mathrm{D}$ est alors conservable et transmissible sous des formats qui autorisent sa manipulation à des degrés plus ou moins avancés.

Cette expérimentation montre que la $3 \mathrm{D}$ peut s'inscrire dans la chaine graphique et donne un aperçu du potentiel analytique qui en découle. L'acquisition $3 \mathrm{D}$ par photogrammétrie s'est révélée souple et adaptée à notre activité. systèmes de numérisation, l'acquisition $3 \mathrm{D}$ par photogrammétrie se révèle la solution couplant le coût d'exploitation le plus faible avec la plage d'utilisation la plus large. Sa limite n'est conditionnée que par les capacités optiques des appareils photographiques, notamment pour les vues rapprochées. Toutefois, cette technique correspond à un mode de relevé qui peut être complété, pour les petites surfaces, par d'autres systèmes d'acquisitions $3 \mathrm{D}$ comme les scanners. Ainsi, la photogrammétrie associée au scanner manuel permet de réaliser l'ensemble des numérisations allant du petit mobilier jusqu'à l'échelle du chantier.

Les modélisations 3D sont à la base de l'analyse du relief par des fonctions mathématiques qui permettent d'en révéler les anomalies. Elles constituent une réplique virtuelle qui supporte tout type d'interventions sans mettre en danger le matériel original. Elles permettent les simulations physiques ou mécaniques sur des vestiges afin d'en dévoiler le fonctionnement, la façon dont ils ont été détruits ou dont ils ont résisté à leur environnement. Toutefois, ces approches supposent un changement de culture de notre part. Concevoir ou envisager notre réflexion en $3 \mathrm{D}$ est un processus intellectuel qui nous fait basculer du concept d'empilement vers celui d'emboitement. Le potentiel d'exploitation en archéologie est loin d'avoir été totalement exploré et se rapprocher de l'univers de l'ingénierie permettrait de mieux l'appréhender.

Lillustration archéologique est porteuse d'informations que les spécialistes savent décrypter mais elle est difficilement exportable telle quelle hors de la communauté scientifique. La représentation des faits par le dessin répondait jusqu'ici à une nécessité imposée par le mode de transmission de l'information qui était exclusivement produit sur des supports de communication en $2 \mathrm{D}$. Pour s'adapter à ce format, le fait archéologique était traduit dans un langage graphique qui devait être connu à la fois par son auteur et par le lecteur. Ce mode de figuration ne permettait alors pas le partage de ces données en direction d'un large public. Les modèles numériques ne doivent donc pas servir uniquement à fournir des vues fixes. L'informatique et les modes de diffusion qui y sont associés doivent permettre de conserver et diffuser cette documentation sans perte de qualité. Elle pourra être ainsi consultable et surtout manipulable pour que chacun puisse se faire sa propre opinion sur les éléments modélisés.

Les modèles numériques sont perçus simplement comme des moulages ou des maquettes et permettent de s'affranchir des conventions de représentation. La $3 \mathrm{D}$ est comprise par tous de façon intuitive en faisant appel à un sens inné et non pas à un acquis culturel : dès qu'un modèle s'anime, en suivant un scénario ou les manipulations d'un observateur, son volume est immédiatement appréhendé. Ce type de relevé représente dès lors un potentiel de diffusion plus large, aussi bien scientifique que grand public.

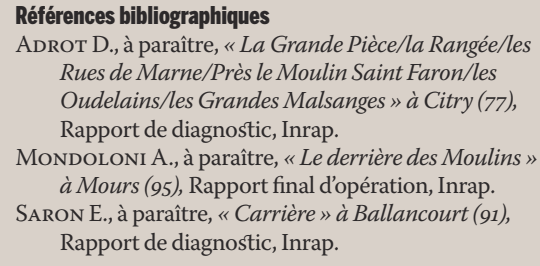

\section{Deux exemples de modélisation en 3D} de sites urbains

Mehdi Belarbi, Inrap

\section{L'enceinte de Philippe Auguste à Paris}

En 2010, une première intervention dans une cour de l'Hôtel des Monnaies, quai de Conti, a mis au jour la face interne de l'enceinte de Philippe Auguste sur une profondeur de $4 \mathrm{~m}^{\mathbf{8}}$. Un deuxième diagnostic, réalisé en 2011, dans la même cour, a dégagé le parement externe de cette enceinte sur une hauteur identique?. L'acquisition 3D par photogrammétrie testée à l'Inrap a permis de modéliser la maçonnerie découverte lors de cette deuxième intervention.

La numérisation a, dans ce contexte, de nombreux avantages. La fouille en puits blindé, effectuée par des puisatiers, ne permet pas une observation globale de la stratigraphie qui est masquée par les boisages mis en place au fur et à mesure du terrassement. Les étaiements, indispensable à la sécurité, sont également des obstacles à l'examen des parois. La $3 \mathrm{D}$ facilite la réalisation de relevés globaux débarrassés des éléments techniques qui en perturbe la lecture et fournit une documentation qui autorise une étude architecturale [ill. 1]. De plus, parce que les photographies numériques prises par le responsable d'opération, lors du premier optimisée si l'on utilis des focales courtes microprocesseurs puissants permettan des calculs rapides.

d'opération Paul Celly, Inrap, 2011.

9. Diagnostic Paul Celly, Inrap, 2010 et 2011 
1 Projection vertical du parement Nord

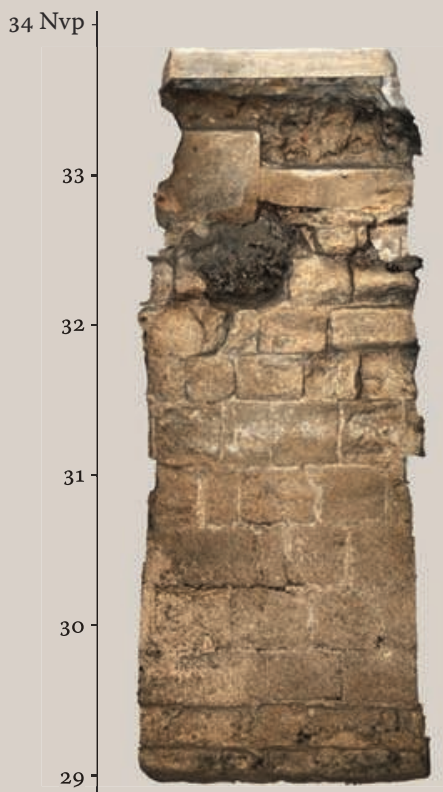

29
Sud

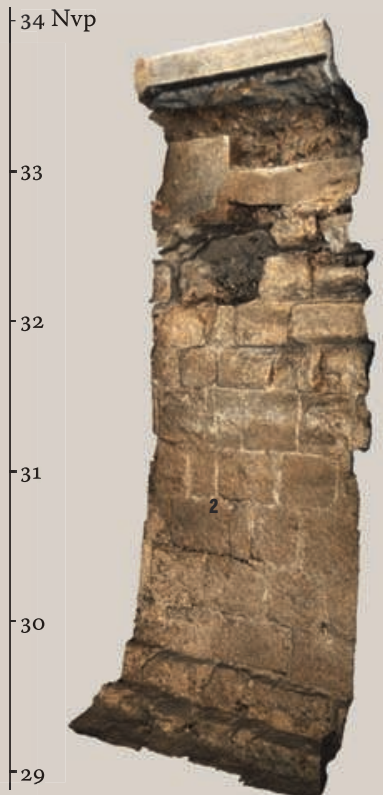

Vue cavalière du parement

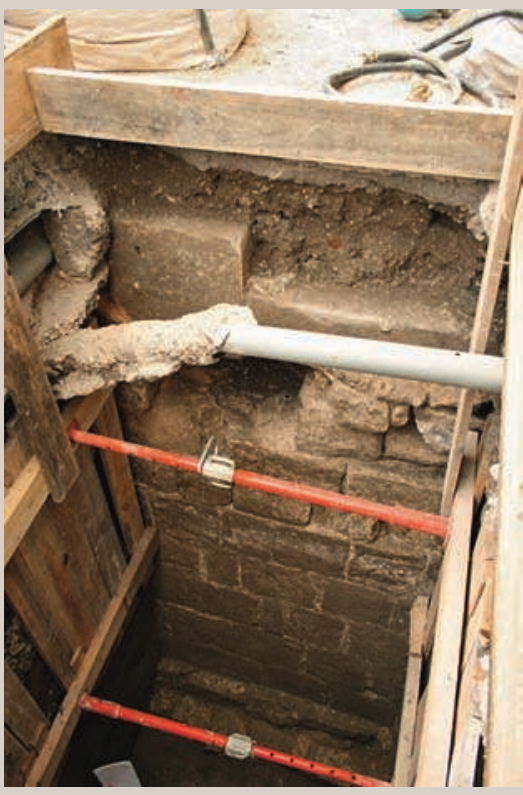

1 Enceinte de Philippe-Auguste dans I'Hôtel de la Monnaie, quai de Conti à Paris. Vue

des contraintes du diagnostic et élévation du mur d'après la modélisation en 3D.

ร
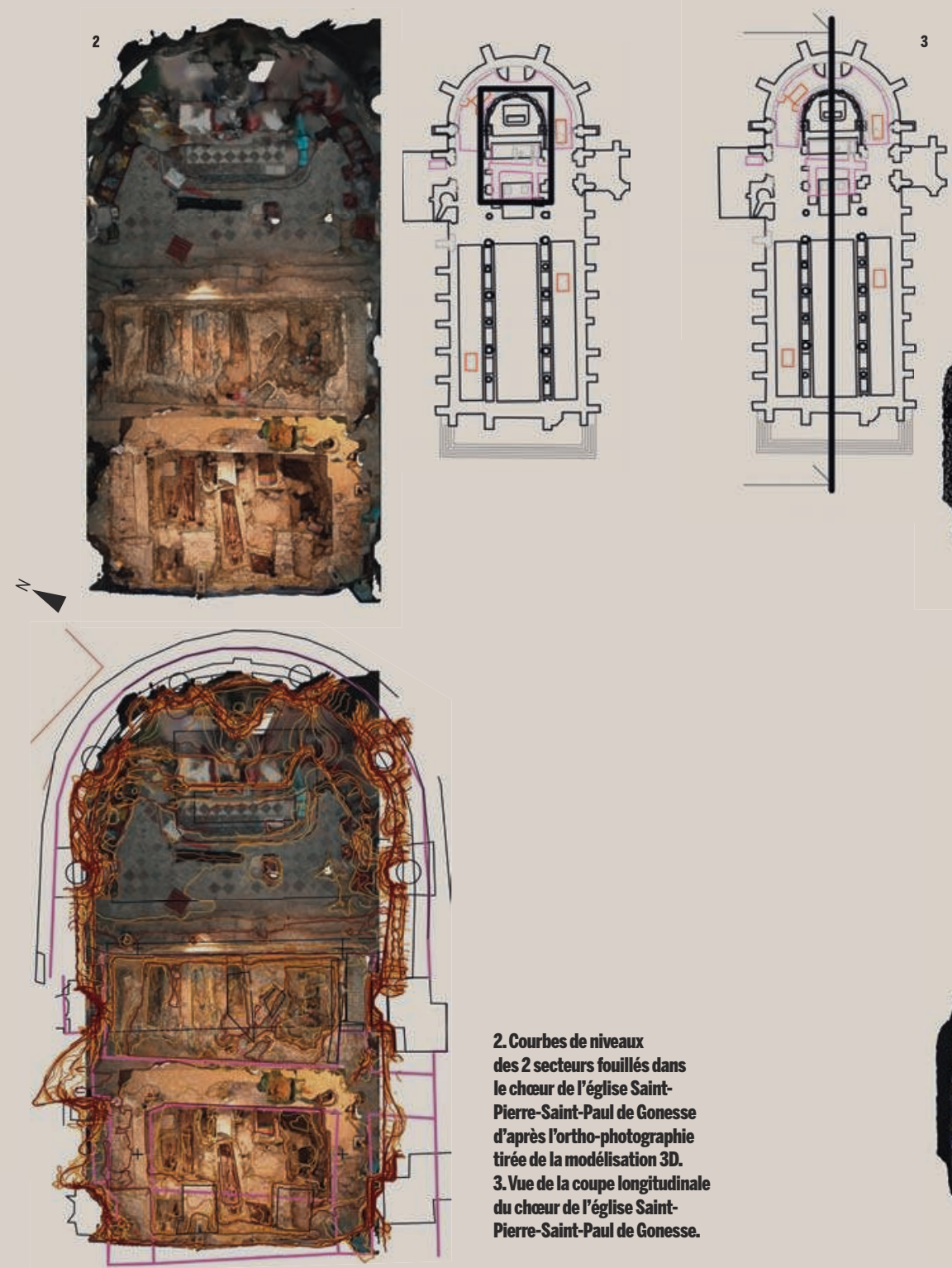

2. Courbes de niveaux des 2 secteurs fouillés dans le chœur de l'église SaintPierre-Saint-Paul de Gonesse d'après l'ortho-photographie tirée de la modélisation 3D. 3.Vue de la coupe longitudinale du chœur de l'église SaintPierre-Saint-Paul de Gonesse.
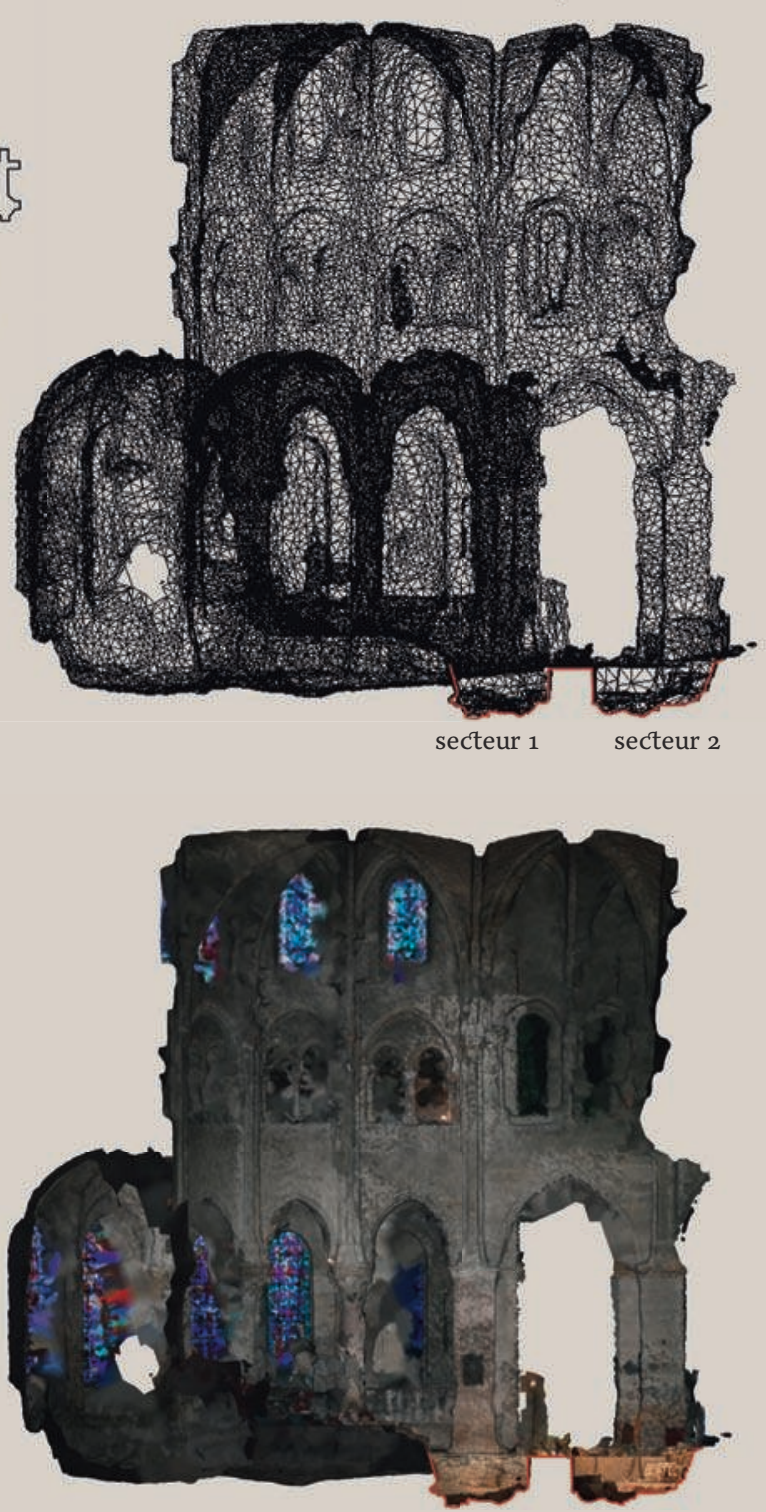
diagnostic, se complétaient, il a été possible de calculer un modèle $3 \mathrm{D}$ de l'intérieur de cette enceinte. La compilation des deux modèles permettra de proposer une vision globale et inédite de cette portion d'enceinte, malgré le décalage de ces interventions dans le temps et dans l'espace. Enfin, ce type de relevé a un intérêt pour la sécurité et pour l'organisation du travail. Il peut être effectué à distance de zones dangereuses à relever manuellement et il limite le temps de présence dans le sondage : de fait, le temps de prise de clichés est plus court que celui d'un relevé traditionnel dessiné, ce qui diminue en conséquence le temps d'attente des terrassiers. Ainsi, ces deux activités alternent avec une plus grande efficacité.

L'église Saint-Pierre-Saint-Paul de Gonesse La fouille de cette église gothique du Val-d'Oise a été effectuée en deux phases ${ }^{1}$. La première eut lieu dans le cœur et le déambulatoire de l'abside. Durant l'été 2012, l'intervention s'étendra à la nef. Un premier modèle numérique a été réalisé à partir de cinq campagnes de topographies et de relevés par photogrammétrie. Ce document restitue un instant « $\mathrm{T}$ » de la fouille, où le principal niveau de sépultures a été dégagé [ill. 2]. Cette opération archéologique se limitant au sous-sol, aucun relevé de bâti n'était prévu. Mais la reconstruction tridimensionnelle a permis un relevé architectural intégrant les voûtes et les différents éléments qui entourent les zones fouillées. Elle a mis en évidence le tracé régulateur sur le chœur de l'église et a permis d'établir la position de son centre théorique 2 . Cette information, essentielle dans ce contexte, facilitera la mise en perspective des fondations découvertes avec l'édifice. Ces relevés peuvent donc être replacés dans le volume entier du chœur. Les moulures des voûtes, des bases de colonnes ou des chapiteaux sont visibles et exploitables pour toutes sortes d'observations. La première phase consistait surtout à fouiller un ensemble de tombes accumulées durant près de dix siècles ; ce type de fouille s'effectue essentiellement à plat, avec prises de données topographiques et altimétriques nombreuses pour conserver un maximum d'informations sur la position des fosses sépulcrales et des squelettes. Aucune berme ne peut aisément être conservée et les relevés en coupe sont rares. La modélisation permet, là encore, de réaliser a posteriori ces profils de façon rapide, précise et pertinente, qu'il s'agisse de relevés entre sépultures ou intégrant le volume général du bâtiment [ill. 3]. De la même façon qu'une photographie générale, ces MNS conservent toutes les informations sur le relief et les volumes des vestiges. Ils constituent ainsi une base de données pour analyser les relations altimétriques des structures et effectuer des mesures dans les trois dimensions. Plus simplement, ils laissent la possibilité de naviguer dans le modèle pour visualiser l'environnement lors de la fouille ou des interventions ultérieures.
Représenter des mobiliers complexes grâce à la $3 \mathrm{D}$

Pascal Raymond, Inrap

Le mobilier lithique de grandes dimensions, lourd et encombrant, est particulièrement complexe à relever. Il doit néanmoins être représenté dans son contexte, notamment par rapport au plan horizontal [ill. 1], mais également en vue géométrale selon sa morphologie. De toutes les méthodes disponibles, la modélisation $3 \mathrm{D}$ paraît la plus appropriée et simplifie la réalisation de cette documentation. Par ailleurs, le souci d'accessibilité des vestiges ne s'explique pas seulement par le caractère destructeur de notre activité, mais parfois par des raisons économiques, légales ou physiques, notamment pour les mégalithes. L'un des intérêts de cette modélisation est également le retour possible à une information initiale d'une grande richesse.

\section{La modélisation de mégalithes gravés} Trois des blocs de grès gravés découverts à l'occasion d'un diagnostic à Citry (Seine-et-Marne) (Adrot, 2012), ont ainsi été modélisés in situ, puisqu'ils ne pouvaient pas être déplacés (surface de $4 \mathrm{~m}^{2}$ et poids de près de 3,5 tonnes pour le plus volumineux). Lors d'un diagnostic, la collecte de l'information doit se faire rapidement. Par ailleurs, l'étude se fondant sur l'échantillonnage, elle doit concilier le besoin d'identification avec la nécessité de ne pas endommager un éventuel site. Enfin, l'étude de ce type de vestiges nécessite des moyens (transport adapté, temps nécessaire au prélèvement et à l'analyse) qui n'ont pu être mis en œuvre dans ce cadre. La nécessité de reporter certaines observations imposait d'autant plus un enregistrement rigoureux de leur géométrie que le projet d'aménagement (carrière) pouvait être annulé ou modifié, ce qui aurait rendus inaccessibles ces blocs durant une période indéterminée. La modélisation $3 \mathrm{D}$ des blocs a donc été utilisée pour l'enregistrement de leur proportion avec un niveau de précision permettant la restitution des principales gravures. Avec une moyenne de 40 photographies par modèle $3 \mathrm{D}$, nous avons produit deux $\mathrm{MNS}^{\mathbf{3}}$ pris sur le terrain et deux autres après nettoyage des parties du bloc 34-15, cassé lors du diagnostic [ill. 2]. Associées à ces relevés, des portions gravées de $0,5 \mathrm{~m}^{2}$ ont fourni une documentation supplémentaire avec une résolution inférieure au millimètre sur ce qui correspond, pour chaque modèle, à un nombre de polygones compris entre 1 et 1,5 million. À partir de ces documents, il est possible de faire ressortir les gravures pour fournir des illustrations préalables à leur étude. La surface de l'un des blocs est représentée ici avec les couleurs enregistrées grâces aux photographies, mais également avec une teinte neutre en faisant varier la source d'éclairage [ill. 3.a, b, c]. Un autre mode de représentation révèle également les 